\title{
DATACIÓN DE UNA VIGA DE LA IGLESIA DE SAN PEDRO DE LA NAVE (ZAMORA)
}

POR

\author{
EDUARDO RODRÍGUEZ TROBAJO \\ Centro de Investigación Forestal, INIA, Madrid \\ FERNÁN ALONSO MATTHIAS \\ Instituto de Química - Física «Rocasolano», CSIC, Madrid
}

LUIS CABALLERO ZOREDA

Centro de Estudios Históricos, CSIC, Madrid

\section{RESUMEN}

Las dudas sobre la adecuada adscripción cultural de las iglesias consideradas de época visigoda, obliga a conseguir dataciones lo más seguras posibles. Por ello se ha realizado este estudio interdisciplinar, que compagina la dendrodatación, la datación por carbono-14 y la arqueología y cuyo resultado data el árbol de pino albar o laricio con el que se talló la viga de la iglesia de S. Pedro de La Nave en una fecha post quem de 330-474 AD (ref. CSIC-1318). Sus caracteres anatómicos nos indican que la secuencia dendrocronológica obtenida de 282 años es completa, por lo que la viga tuvo que ser reutilizada, como debió ocurrir al menos con parte del material de construcción. Los análisis han servido para ensayar la aplicación de esta metodología a las iglesias altomedievales españolas y mejorarla para lograr resultados más fiables y precisos.

\section{SUMMARY}

Churches usually ascribed to the Visigothic period are difficult to place in a clear cultural framework, with the result that other dating methods assume a vital importance. In this study, dendro-chronology, carbon-14 dating, and archaeological evidence combine to give a terminus post quem of AD 330-474 for the pine-trunk employed for the beam of the church of San Pedro de la Nave ("pino albar o laricio"). Given the trunk's completed dendro-chronological sequence of 282 years, the beam in its present location must constitute a case of re-use. At least some of the other elements employed in the construction of the building must also have been re-used. The discussion serves to illustrate how an interdisciplinary approach can be applied to the study of early medieval Spanish churches and how it might be refined to produce ever more reliable and valuable results.

\section{INTRODUCCIÓN}

Está admitido que las denominadas iglesias de época visigoda no poseen elementos de datación absoluta, por lo que su adscripción cronológica se efectúa fundamentalmente mediante argumentos de carácter tipológico y estilístico, lo que hace que no exista un consenso unánime sobre su cronología. Recientemente se ha reabierto la controversia sobre ellas, planteándose la hipótesis de que sus caracte- rísticas formales, constructivas y decorativas deriven de técnicas aportadas por la cultura musulmana tras la imposición en la Península del omeya 'Abd al-Rahmân I, o sea en una fecha entre uno y dos siglos posterior a la visigoda de la segunda mitad del s. VII (Caballero 1994-95 y 1997).

La conveniencia de buscar pruebas alternativas de índole cronológica nos decidió a iniciar una línea de análisis dendrocronológico y de carbono-14 de las relativamente abundantes maderas que forman parte como elementos constructivos de nuestras iglesias alto medievales. Como paso previo se planteó un experimento aislado que permitiera, a partir de su experiencia, poder elaborar un proyecto más amplio con suficientes garantías '. Para ello se buscó una madera cuya cronología fuera significativa en el contexto arquitectónico del que formara parte, que poseyera alta probabilidad de ofrecer un resultado aceptable y que fuera fácilmente accesible. Así se eligió la viga de la iglesia de S. Pedro de La Nave (Zamora). Su procedencia y relación con el edificio es seguro, como se había contrastado recientemente (Caballero y Arce 1997: 246, fig.7 y 8); sus caracteres de especie y cantidad de madera son adecuadas y, finalmente, está depositada en un $\mathrm{Mu}$ seo lo que permite eludir los problemas que plantea intervenir en una madera inserta en un monumento.

El análisis propuesto está de acuerdo con los principios metodológicos de la Arqueología de la Arquitectura, de modo que forma parte de la resolución de una problemática histórica previamente

1 Agradecemos la imprescindible ayuda que nos prestaron para la realización del trabajo $\mathrm{D}^{\mathrm{a}}$ Rosario García Rozas, directora del Museo de Zamora, a quien se debe la fig. $2 ; \mathrm{D}^{\mathrm{a}}$ Hortensia Larrén Izquierdo, arqueóloga territorial de Zamora; Dr. D Enrique Nuere Matauco, arquitecto. Los trabajos se efectuaron con autorización de la Consejería de Educación y Cultura de la Junta de Castilla y León y dentro del proyecto de investigación Las iglesias de Repoblación. Documentación y análisis, DGICYT. PB 94-0062. 
planteada y se incardina con otras estrategias de investigación, en este caso con la estratigráfica (Parenti 1988). Además, desde un principio, se planteó como un trabajo pluridisciplinar que no sólo sirviera a la resolución histórica, sino en la misma medida a los otros dos campos científicos implicados.

La datación por métodos empíricos de maderas altomedievales españolas encuentra en la actualidad algunos condicionantes importantes. Por una parte, no es posible la dendrodatación absoluta al carecerse de cronologías de referencia para la Península Ibérica que superen los 1.000 años de antigüedad. Esta situación es debida a la escasez de elementos de madera y su dispersión en una amplia área geográfica que, al estar constituidos por especies propias de cada región, forman un conjunto heterogéneo de difícil interconexión. El escaso pero uniforme grupo de maderas altomedievales abre la posibilidad de ampliar las secuencias dendrocronológicas del centro de la Península más allá del s. XI. Por otra parte, la datación mediante el carbono-14 tiene un papel relevante al poder aportar una cronología absoluta a los elementos lígneos altomedievales. Existen numerosos precedentes de dataciones combinadas entre ambos métodos (Gruppo di Lavoro 1985), que han permitido desarrollar interesantes estudios de cronología relativa, tanto entre fases de la misma construcción, como entre enclaves diferentes. La datación de estas secuencias parciales por carbono-14 permite situarlas temporalmente, aunque con márgenes de fluctuación ineludibles mientras no se unan en una secuencia común.

Un aspecto que singulariza el presente trabajo es el intento de mejorar la aplicación de ambas técnicas con el fin de lograr resultados más fiables y precisos. Consideramos que la aplicación de este plan metodológico puede rendir excelentes resultados de cronología relativa y, a la postre, hará posible la ampliación de las cronologías de referencia necesarias para lograr dataciones de mayor precisión.

\section{EL MATERIAL}

Son muy escasos los datos que tenemos sobre los restos de madera descubiertos durante las labores de desmonte de esta iglesia, efectuado bajo la dirección del arquitecto Alejandro Ferrant entre los años 1931 y 1932. La primera referencia es la de Torres Balbás (p. 130), que presenció los trabajos y conoció los informes y noticias directamente de sus responsables. La importancia de esta referencia es que se hace al valorar la restauración un año después de terminarla, aunque sus detalles no dejan de

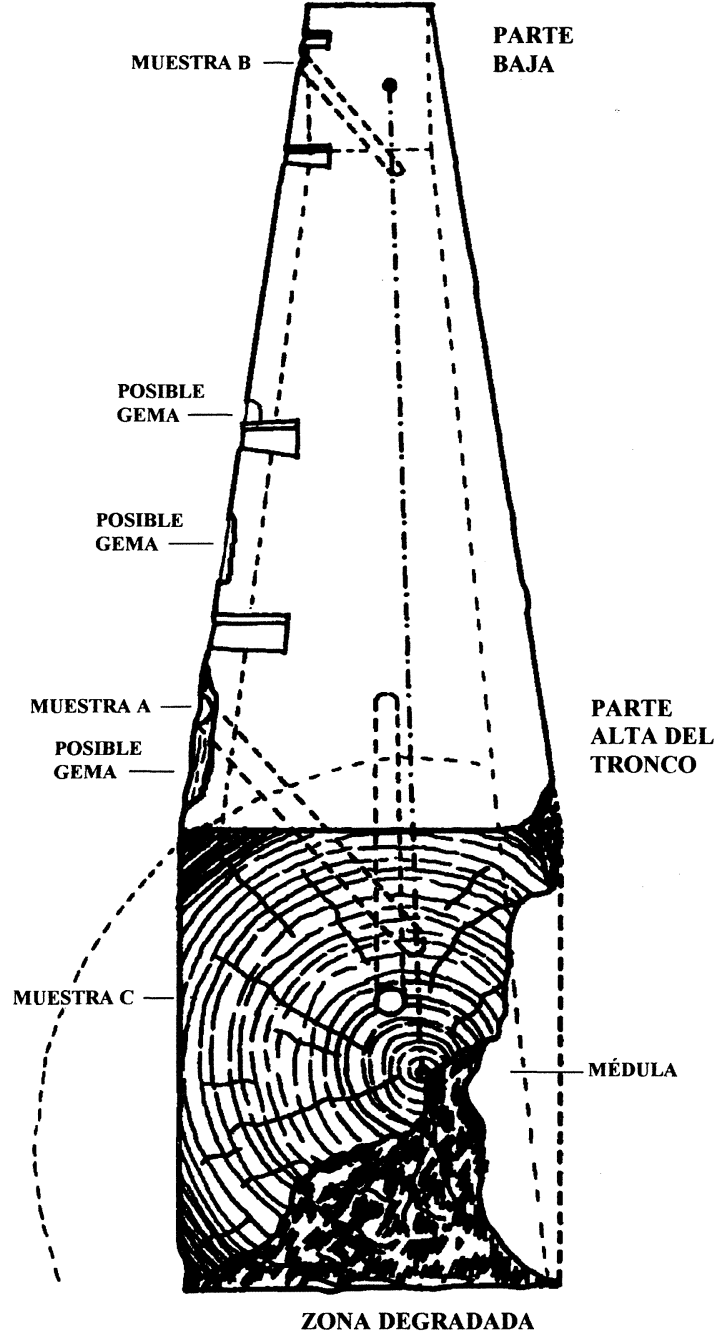

Fig. 1.-Esquema de la viga y situación en ella de las calas.

plantear dudas. Describe cómo algunos de los sillares tenían grapas de madera en forma de cola de milano, embutidas en sus cajas talladas, cogiendo dos o tres (sillares) y con una longitud hasta de 70 $\mathrm{cm}$. Cita también el hallazgo sobre el arco triunfal..., empotrada en el muro, (de) una viga horizontal que apeaba la parte situada encima de él.

Camps Cazorla (p. 607), testigo del traslado, efectúa el primer análisis científico de la iglesia tras su nueva ubicación, en 1940. Se refiere a las grapas y a la viga como particularidades ingeniosas de su técnica constructiva. Pero la cita es brevísima y sólo añade la explicación de la finalidad de la viga por encima del arco toral, metida dentro del aparejo como elemento de trabazón de la estructura del edificio y no simplemente como elemento de descarga. 
Gómez Moreno, Director General de Bellas Artes en el momento del traslado, se refiere en 1966 a las maderas aparecidas en La Nave: ... encajar grapas de madera de doble cola entre los sillares más oprimidos, especialmente en la cuarta hilada, y no sólo ello, sino que se ligaron varios con otra larguísima y se descargó el arco de la capilla metiendo encima una robusta viga de roble, publicando una planta con las grapas de la cuarta hilada que, lógicamente, obtendría por intermedio de Ferrant (Gómez Moreno 1966: 128, lám. IX). Corzo (p. 53) analiza con detalle este plano llegando a la conclusión de que debe considerarse una planta ideal que quizás mezcla datos de varias hiladas distintas. La grapa larguísima debe ser la que en ese plano une tres sillares en la esquina SE. del ábside. Acudiendo a la dimensión real de los sillares grapados, la longitud de la grapa estaría entre 60 y $70 \mathrm{~cm}$. De este modo coinciden las observaciones de Gómez Moreno y de Torres y se puede asegurar que al menos una grapa de esta dimensión unía dos o tres sillares ${ }^{2}$. Es contradictoria la referencia al tipo de madera de la viga, según Gómez Moreno de roble, cuando según Corzo sería de pino.

Mateos Rodríguez en 1980 (Mateos y Esteban: 28) cita otra pieza más de madera, una caja de reliquias aparecida en el interior del altar moderno de la iglesia. La noticia la recoge de la prensa local, El Heraldo o El Correo de Zamora de 1930. Del resumen que hace queda en duda su ubicación exacta, posiblemente en el loculus o sepulcrum situado en la parte superior del ara en forma de pilastra que sostendría el tablero y que se encontraba dentro del altar macizo moderno. Estaba construida en madera de retablo del siglo XVIII y en su interior se encontró, además de las reliquias, un acta con la fecha de 1854 .

Los datos para nosotros de mayor interés los ofrece la monografía de Corzo (p. 53-4 y 84-5). Documenta los lugares donde hoy se pueden ver encajes en los sillares para la colocación de grapas; ofrece la lista que de estos encajes conservaba Gómez Moreno, con los datos de Ferrant, por la que sabemos que aparecieron más de un centenar, número que se incrementaría con los de las hiladas superiores desaparecidas; recuerda que todas las grapas, excepto las dos conservadas, desaparecieron quemadas en una hoguera de los obreros y que se conservó otra tercera en la colección de Gómez Moreno,

${ }^{2}$ Hauschild (1972) publica el plano de Gómez Moreno corrigiendo el detalle de esta grapa que, según su plano, sólo sujetaría dos sillares. Quizás Hauschild, al copiar el plano de Gómez Moreno, aprovechó para corregir un detalle -la excesiva longitud de la grapa- que al no comprenderlo consideró un error del plano de Gómez Moreno. quien la tenía encima de su mesa de trabajo como pisapapeles. La documentación gráfica de Alfonso Jiménez que acompaña el trabajo de Corzo confirma la situación de Ferrant (Corzo: pl. XVI, sección $\mathrm{C})$, que luego nosotros volvimos a documentar (Caballero y Arce: fig. 7 y 8). Por otra parte, su utilización coetánea a la construcción de la iglesia se confirmaría por la existencia de los encajes para grapas que tiene tallados. Finalmente, Corzo añade otra pieza más, un fragmento de viga de armadura de la cubierta medieval, también procedente del traslado efectuado por Ferrant (Corzo: 54 y 190).

En la actualidad, en el Museo de Zamora se conservan cuatro de estas piezas procedentes de la iglesia de La Nave. Sus datos son los siguientes:

1) número de inventario 150 , viga de madera de pino. Según el inventario manuscrito del Museo, redactado por su director V. Velasco: sacada del muro que separaba la nave de la capilla absidal de S. Pedro de La Nave... en cuya parte superior estaba empotrada sirviendo de umbral por la parte interior de la puerta de ingreso al recinto que lleva sobre dicha capilla absidal y, por tanto, de su época. Longitud 278 y escuadría $30 \times 38 \mathrm{~cm}$, con cuatro encajes en forma de cola de milano, situados en la misma cara y arista, una pareja a $16 / 21$ y $73 / 79 \mathrm{~cm}$ de su extremo más cercano y la otra a 40/46 y 93,5/99 cm del extremo contrario, con dimensiones entre $5 / 6,5 \mathrm{~cm}$ en la boca, 7.5/9 en el fondo y 11/19 de largo.

2) núm. inv. 151, viga de madera de pino. Par de techumbre vista y preparada para sostener tabicas. Tardomedieval o posterior. Según el inventario de V. Velasco: armadura del tejado de la casa rectoral de la iglesia de S. Pedro de La Nave. Posee dos aristas biseladas y, en uno de sus extremos, dos acanaladuras oblicuas a su eje, entre las que se dispone un orificio circular de $25 \mathrm{~mm}$ paralelo a las anteriores atravesándola. Restos de policromía. Longitud 120 y escuadría $21 \times 17 \mathrm{~cm}$.

3 y 4) núm. inv. 152, 1 y 2 , dos grapas de madera de encina. Para sillería, con forma de doble cola de milano. Longitud 39 y 38,5, ancho máximo 8,2 y 10,2 , grueso 3,3 y $3,1 \mathrm{~cm}$.

Nuestro trabajo se centra en la viga descrita en primer lugar. Como hemos dicho, su lugar de aparición está bien contrastado, encima del arco de triunfo, en una caja situada en el muro donde está abierto el arco de triunfo, separada $33 \mathrm{~cm}$ de su paramento occidental y formando el umbral de la puerta o ventana de acceso a la habitación situada encima del ábside, hoy cerrada. Sus cuatro encajes corresponden, con casi absoluta verosimilitud, a grapas de unión con los sillares que se situarían, lógicamente, en la cara superior de la viga. 


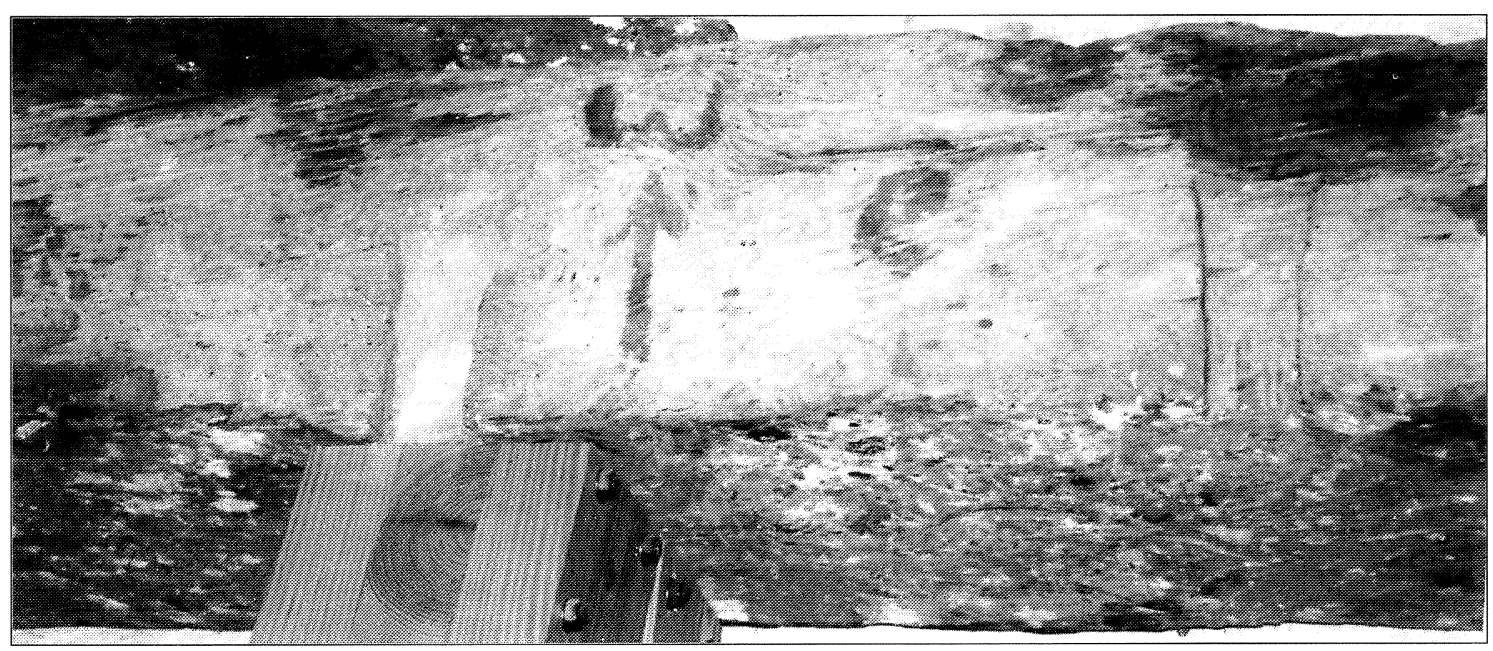

Fig. 2.-Algunos de los signos pintados y dos encajes de grapas.

La viga presenta signos realizados con pintura roja, una flecha apuntando a una especie de omega, el número (?)574, una letra «e» y gotas de pintura. Los signos coinciden con los observados en otros sillares de la iglesia y consideramos corresponden a las marcas efectuadas en los sillares y elementos de la iglesia durante su traslado, de modo que el número pudo corresponder a la numeración seguida de los elementos trasladados. La flecha debió marcar una orientación geográfica, quizás el Este si la omega fuera en realidad una E tumbada. De ser así, la cara marcada sería la visible al desmontar las hiladas y por lo tanto la superior, correspondiendo efectivamente con la de las cajas para grapas (Caballero y Arce: 256, corregimos la lectura, quizás una e minúscula mejor que una $\mathrm{C}$ mayúscula).

La cara opuesta a la del enganche de los engatillados presenta una intensa pudrición, lo que hizo pensar que, de haber sufrido una humedad prolongada cuando se encontraba en el edificio, tenía que ser la inferior o la superior. Pero la situación de las marcas con pintura roja y la dificultad de que se hubiera podrido tan uniforme y profundamente dentro del muro, hace más verosímil que esta consunción ocurriera una vez recuperada la viga ${ }^{3}$.

La viga hubo de servir para atar el muro de testero de la nave de anteábside, de modo que no se abriera lateralmente partiendo el dintel de la ventana y abriendo las dovelas del arco de triunfo. En

${ }^{3}$ Agradecemos a D. José Alonso Luengo, REARASA, Zamora, la discusión sobre estos extremos, así como la observación sobre las medidas de la viga, media vara de ancho, y su talla, similar a la de los sillares, con destral de $5-6 \mathrm{~cm}$ de huella, y la de los engatillados con hacha y no con formón, datos todos ellos que considera arcaicos. concreto debía encadenar los sillares correspondientes a la hilada de umbral de la ventana: las dos grapas interiores unirían, a los lados de las jambas, con el largo sillar que hace de umbral y las dos exteriores unirían con el centro aproximado de los sillares siguientes. Según Cámara (Caballero y Arce: 268) actuaba como tirante de los empujes de la bóveda superpuesta, que en aquel punto podían tender a abrir el muro debilitado por la ventana de acceso a la habitación superior y por el arco de triunfo, y no de descarga de los pesos que seguirían gravitando sobre el arco. El sistema comprendía, de arriba a abajo, el dintel tallado en forma de arco, la viga que encadenaba los tres sillares, el sillar adovelado encima de la clave del arco de triunfo, que actúa como descarga, y este arco.

\section{ESTRATEGIA DEL MUESTREO}

La toma de muestras ha sido diseñada con el objetivo de alcanzar una cierta precisión y fiabilidad en los resultados del estudio dendrocronológico y del carbono-14. De este modo, se han considerado algunos factores relevantes que pueden afectar a la calidad de los ensayos.

En la datación por carbono-14, la medición de la actividad será tanto más exacta cuanto mejor se haya conservado a lo largo del tiempo la muestra original. Los riesgos de contaminación aconsejan la elección de partes bien conservadas, tanto para muestras de tamaño normal como para aquellas que por su abundancia permiten aplicar un tratamiento químico de extracción de celulosa pura (Aitken, 1990). Es también importante que la muestra esté 
integrada por un número acotado de anillos con el fin de que la edad del carbono-14 sea calibrada adecuadamente y pueda ser referida a un intervalo bien localizado dentro de la secuencia de anillos del árbol.

Por su parte, la dendrodatación mejora su precisión si las secuencias de anillos son lo más completas posibles (idealmente, hasta la gema o corteza del árbol), pues de este modo resultará un terminus post quem más ajustado (Eckstein et al., 1984). Asimismo, el control de anomalías es esencial para la viabilidad de la propia dendrodatación, lo que aconseja repetir el muestreo en distintas partes de cada elemento.

La extracción de las muestras para cada uno de estos análisis requiere operar en direcciones perpendiculares entre sí, axial y radial. Como es evidente, pueden presentarse dificultades en esta operación, dependiendo de la forma y accesibilidad de la pieza de madera. Además, el muestreo deberá ser practicado de forma que resulte lo menos lesivo posible para el material.

En la figura 1 se muestra en esquema la extracción de las tres muestras o calas de la viga: las calas A y B se tomaron en dirección radial con el fin de obtener largas secuencias de anillos, mientras que la muestra $C$, destinada a carbono-14, tiene dirección axial. Las calas A y B se practicaron en los extremos para que la secuencia conjunta resultara más larga que las individuales, a la par que se disminuía el riesgo de que ambas muestras estuvieran afectadas por las mismas anomalías. En consecuencia, estas muestras serían aptas para el estudio de la secuencia de anillos (dendrodatación) y, al mismo tiempo, permitirían una localización precisa de la muestra de carbono-14 dentro de la estructura del leño.

En la operación se han utilizado barrenas huecas de $25 \mathrm{~mm}$ de diámetro total que extraen calas de 10 mm de diámetro. Tal como muestra la figura 3 , se tomó la cala $\mathrm{C}$ en una zona interna, sin biodegradación aparente, con anillos de mayor espesor que en las zonas externas de modo que contuviera un corto número de anillos $(<25)$. Se eliminó el tramo más externo, afectado de pudrición y galerías de xilófagos, y se recogió el serrín emergente que junto con la cala central se utilizarían en la radiodatación. Esta perforación permitió la extracción de una muestra suficientemente grande sin provocar un daño notorio a la viga.

A continuación, se practicó el orificio A en una discontinuidad de la arista, que parece corresponder a una zona de gema, hasta interceptar el orificio de la muestra C, si bien la perforación se prolongó has-

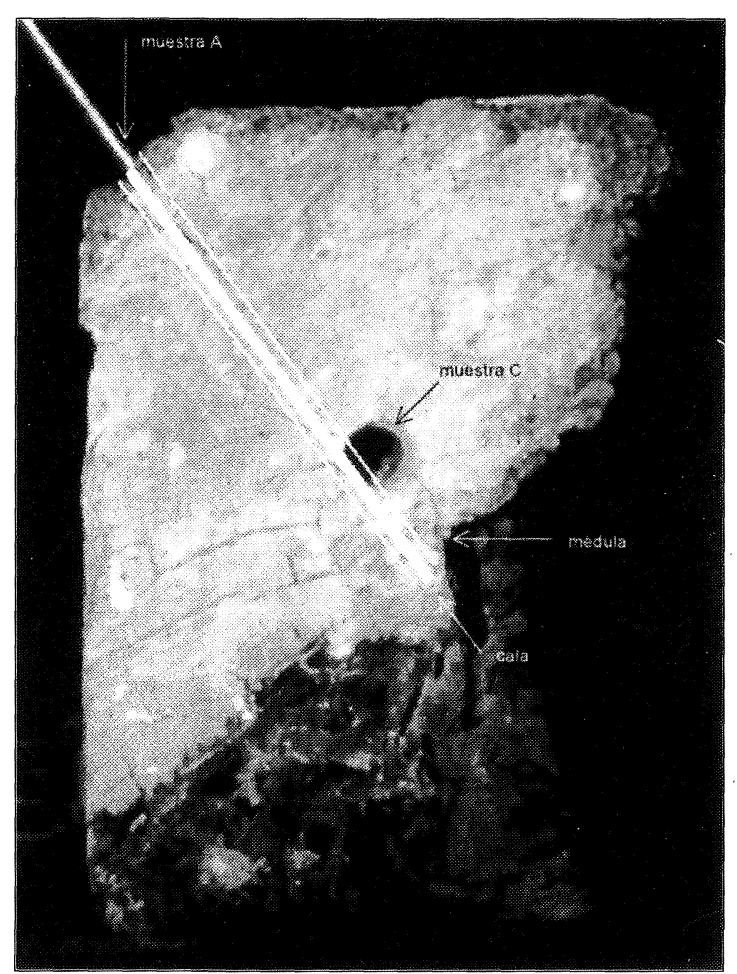

Fig. 3.-Disposición relativa de las calas A (dendrocronología) y C (carbono-14) en la testa de la viga.

ta sobrepasar la médula para que la secuencia fuera más completa (fig. 3). La segunda extracción radial (B) se realizó en la misma arista, pero en el extremo opuesto de la viga.

Finalmente, debemos advertir que en las perforaciones radiales A y B sólo se aprovecharon las calas, mientras que el serrín circundante fue desechado por estar compuesto de madera de todas las edades de la secuencia ( $>250$ años).

\section{RESULTADOS Y DISCUSIÓN}

\section{Estudio dendrocronológico}

Entre las características constitutivas del material debemos destacar la especie de la madera: $P i$ nus sylvestris L. / Pinus nigra Lamb. (pino albar / pino laricio). Si bien no podemos identificar, a partir de la anatomía del leño, a qué especie pertenece realmente la madera, este resultado es de por sí notable pues ninguna de las dos especies tiene en la actualidad una distribución próxima al enclave del edificio. Un resultado más plausible hubiera sido una especie propia de esta región bioclimática como es el caso de los pinos pinaster o piñonero. Pode- 


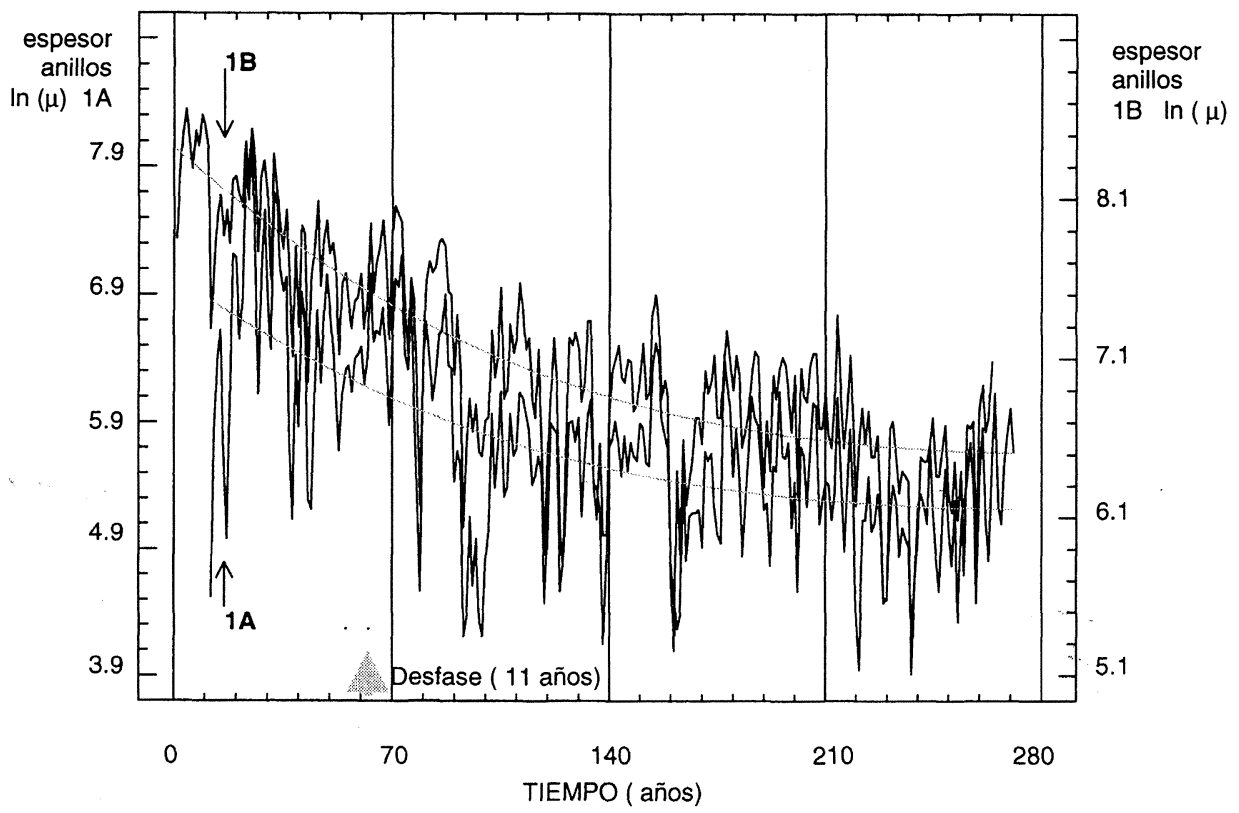

Fig. 4.-Representación de las series 1A y $1 \mathrm{~B}$ (ordenada: logaritmo del espesor de anillo) y sus respectivas curvas de tendencia de la edad.

mos afirmar que, salvo en el caso de que haya existido en sus proximidades una pequeña masa relicta, hoy desaparecida, este material tuvo que ser transportado desde una zona distante cuando menos 100 km (áreas relictas del alto Porma y de Cuellar ${ }^{4}$ ) o más de 200 Km (Sistema Central/Sierra de Urbión); todas ellas regiones actuales de procedencia del pino albar y laricio (Catalán et al., 1991).

La viga ha sido labrada a partir de un grueso árbol de más de 1,50 $\mathrm{m}$ de perímetro y, puesto que la médula se sitúa próxima a una de las caras, pudo extraerse del mismo tronco otra u otras piezas. En cualquier caso, el despiezo fue esmerado como atestiguan sus aristas bien escuadradas. Un origen bien distinto tiene el acusado alabeo de sus caras producido al secarse la madera tras el labrado de la viga en verde, y cuya causa es el fenómeno de fibra revirada que afectó al tronco del árbol.

Otra característica significativa es la presencia de gruesos nudos que corresponden a una fuerte ramificación en las partes bajas del tronco. Se trata de un dato que nos sugiere condiciones de crecimiento sin competencia, en un entorno despejado de otros árboles. Debemos resaltar también la abundancia de

\footnotetext{
${ }^{4}$ La masa forestal espontánea más próxima se encuentra a $145 \mathrm{~km}$ en Cuéllar (Segovia), donde coexisten pinos albares y laricios en una cota de $800 \mathrm{~m}$, tal como ha estudiado Ruíz del Castillo (comunicación personal). Agradecemos también a Ricardo Alía, genetista forestal, sus comentarios acerca de la distribución actual de estas especies.
}

resina en todo el leño que adquiere así un acusado tono rojizo por debajo de su apariencia externa gris; lo cual pudo inducir a Gómez Moreno (1966) a identificar erróneamente este madero como una viga de roble. El predominio de condiciones climáticas extremas es la causa más probable de este incremento de secreción que, por otra parte, ha servido para mejorar la resistencia de la viga a la biodegradación.

En el estudio dendrocronológico sensu strictu debemos destacar, en primer término, algunos parámetros globales como el pequeño crecimiento medio de sus anillos $(0,899 \mathrm{~mm})$ y la acusada sensibilidad media $(0,327)$. Ambos datos refuerzan la hipótesis de que el árbol se desarrolló en condiciones ambientales bastante rigurosas. En la figura 4 se muestran las series 1A y 1B (logaritmo del espesor) y sus respectivas curvas de tendencia (polinómica cúbica), que se estabilizan en torno a los 325 años de edad del árbol. La tendencia es más acusada en la serie 1B (64\% de la variación total), mientras en $1 \mathrm{~A}$ el efecto es mucho menor ( $37 \%)$. Esto es debido a que $1 \mathrm{~B}$ corresponde a la base del tronco $\mathrm{y}$, por consiguiente, se observa un desfase inicial de 11 años debido a la diferencia de edad entre los puntos A y B. Podemos estimar que el crecimiento en altu-

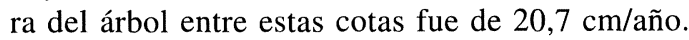

Una vez eliminada la tendencia, podemos apreciar la variación cíclica e interanual (fig. 5) que centra nuestro interés por contener una mayor informa- 
ción del entorno. Las varianzas casi iguales y los altos valores de autocorrelación son datos indicativos de la alta similitud que existe entre las series. En la figura 5 se observa un tramo irregular (93160) con fuertes caídas del crecimiento en los extremos y puntos intermedios. Al no disponer de un conjunto de series de la misma localidad no es posible discriminar si estos mínimos son efectos individuales del árbol o comunes a la masa. Por este motivo, se ha optado por ensayar un ajuste polinómico cúbico únicamente sobre los ciclos de mayor amplitud. A más largo plazo, en la medida que este material sea sincronizado con otros de similar cronología y procedencia, se podrán verificar si estos ciclos plurianuales tienen especial significación en el estudio de la procedencia de la madera.

En resumen, podemos evaluar globalmente el crecimiento de este árbol como el propio de un ejemplar de pino albar/laricio de longevidad apreciable (> 280 años), con variaciones bruscas en el espesor de sus anillos debido a unas condiciones climáticas bastante extremas. Asimismo, su perfil de crecimiento refuerza la hipótesis de que fue un árbol que creció aislado, al menos en buena parte de su dilatada vida. Como consecuencia de lo anterior, es también probable que los ciclos indicados obedezcan más a una respuesta ecofisiológica común de ámbito local que a simples efectos individuales o de competencia.

El último aspecto importante en el estudio del material con fines de datación es la detección de anomalías del crecimiento. En un árbol longevo y creciendo en condiciones limitantes, cabe esperar que en los años más críticos el árbol no llegue a desarrollar, parcial o totalmente, el anillo correspondiente. Hemos podido localizar hasta 7 anillos ausentes: 6 en la serie B y 1 en la serie A (fig. 5). Esta desproporción debe considerarse normal ya que, tal como hemos indicado, la serie B procede de la base del tronco que es más proclive a la aparición de

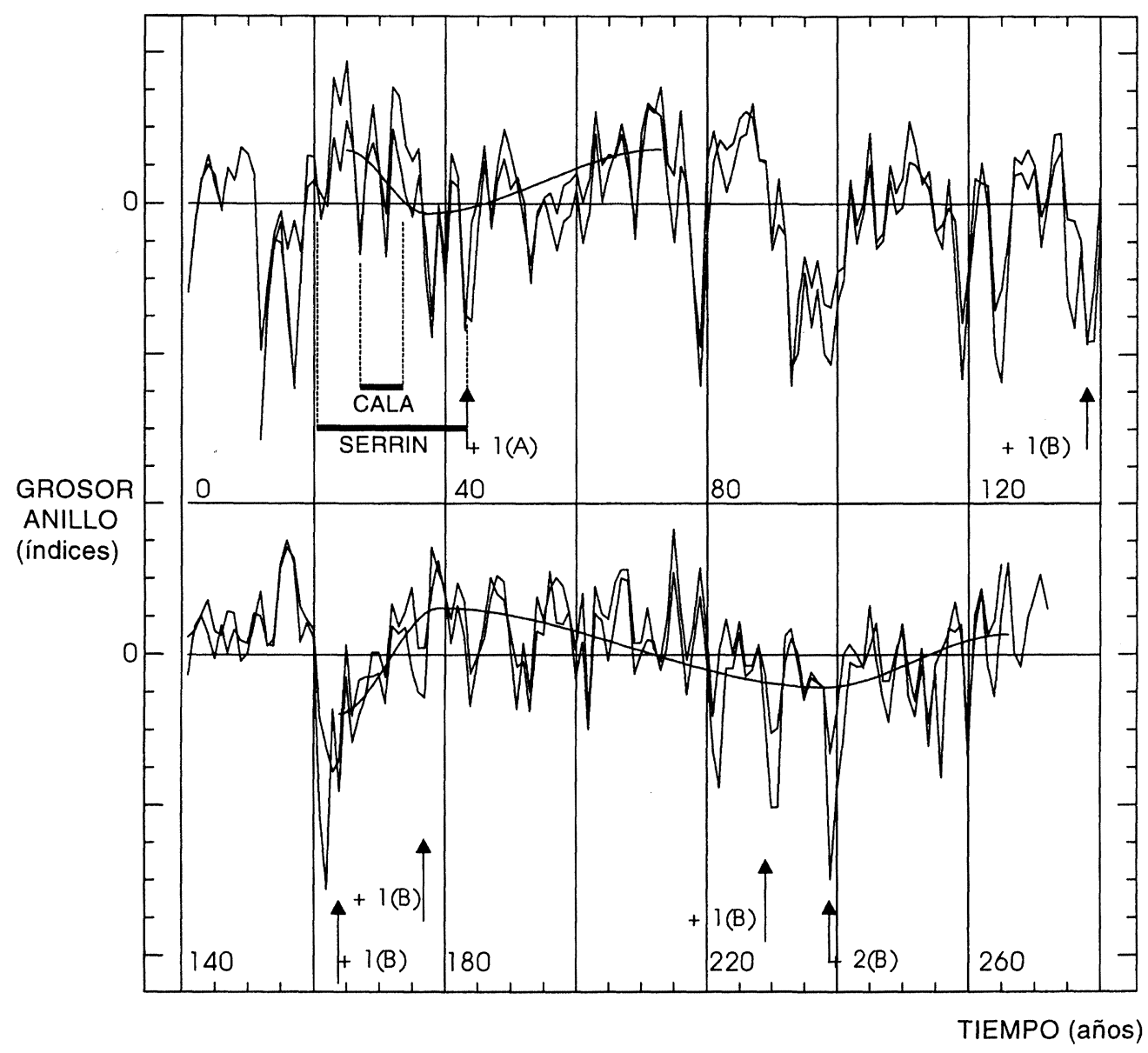

Fig. 5.- Representación de las series 1A y 1B, una vez extraída la tendencia de edad, y curvas significativas de la variación plurianual. 


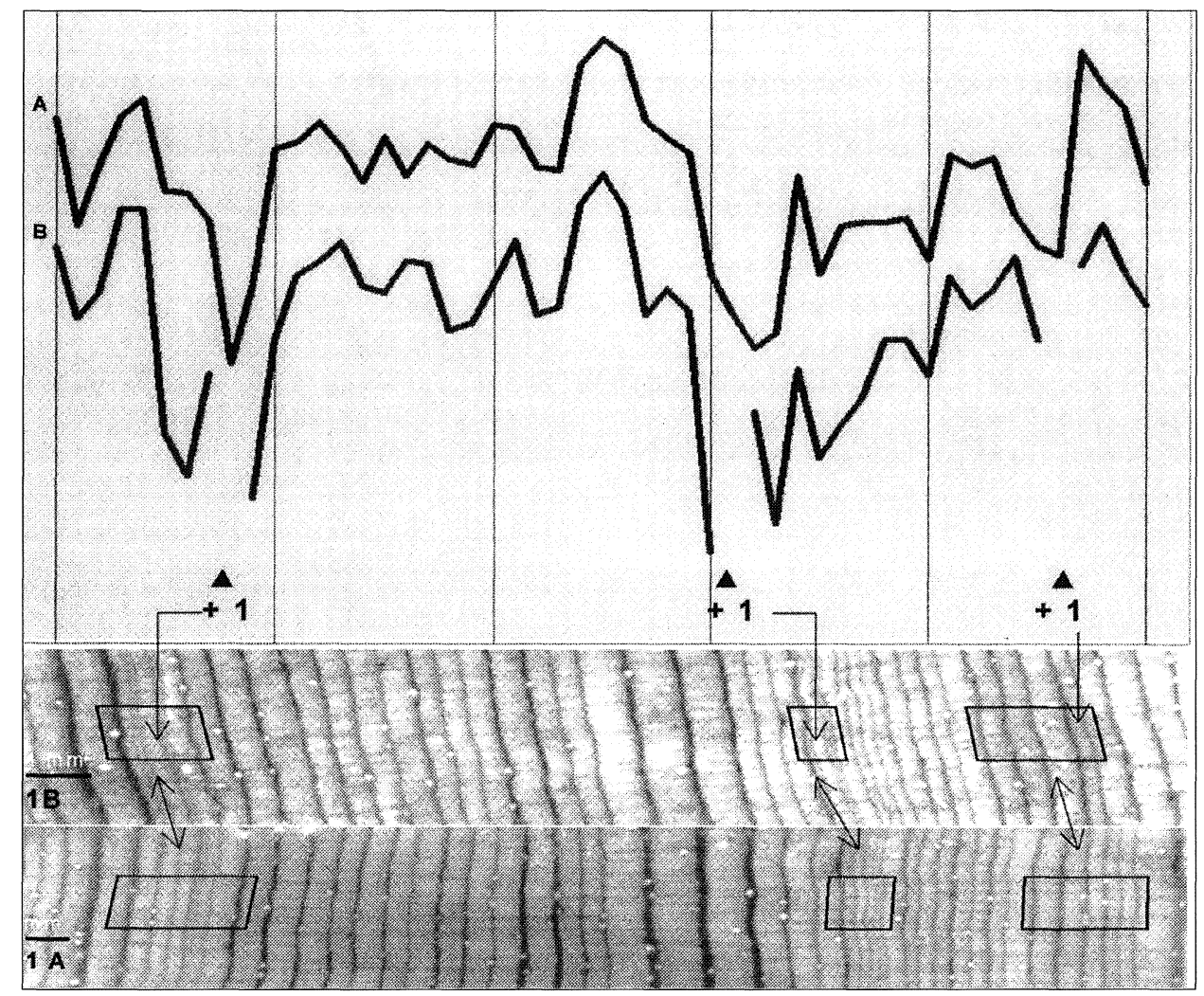

Fig. 6.-La serie 1B presenta la falta de varios anillos que no llegaron a formarse pero que sí aparecen en la serie 1A. La localización de estas anomalías se facilita al comparar las pautas de textura de ambas series.

anomalías. En la figura 6 se observa cómo la serie 1B precisa la «adición» de tres anillos separados 23 y 15 años entre sí. La localización de estos fallos se realiza comparando el perfil de ambas curvas, así como cotejando las pautas de textura entre las series. La tipología más frecuente de esta anomalía se muestra en la figura 7: un anillo muy fino, con su madera final de tan sólo una o dos células, llega a ser «absorbido» por la madera final del anillo precedente.

Existe la posibilidad de que existan también anomalías simultáneas en 1A y 1B que no detectamos al no poder sincronizar con series extraídas de otros árboles. No obstante, se deben estimar en número inferior al de fallos simples (1 ó 2, a lo sumo), si bien en el caso que nos ocupa, dado que la única anomalía que afecta a la serie $1 \mathrm{~A}$ está en zona de crecimiento medio, los fallos parecen ligados al factor ya indicado de la posición de 1B en la base del tronco.

Finalmente, consideremos el terminus post quem resultante del muestreo. La serie 1A está situada en una zona de arista redondeada que podría corresponder a la gema (último anillo del árbol), por lo que la secuencia obtenida sería completa. Tal como se indica en la figura 1, existen dos zonas más de posible gema, que se encuentran en la misma arista y en la mitad de la viga que corresponde a la parte alta del tronco. Esto es coherente con el proceso de labrado al hilo, en el que se elimina progresivamente más madera hacia la base del tronco. Otro dato adicional es el descentrado de la médula en la viga, que nos confirma que el labrado de la arista fue bastante apurado.

Por otra parte, a lo largo de esta mitad de la viga, se aprecian galerías de pequeño diámetro que se caracterizan por afectar sólo a una estrecha banda de uno o dos centímetros de profundidad desde la corteza. Precisamente, la abundancia de estas galerías fue causa de la fractura de la cala $1 \mathrm{~A}$, separándose un fragmento externo de 11 anillos muy degradado, por lo que es posible que entre ambos fragmentos se hayan podido perder uno o dos anillos.

Los datos anteriores indican por sí mismos la existencia de gema en $1 \mathrm{~A}$, o al menos en su proximidad, con un margen inferior a una década. Sin embargo, el estudio de la disposición de los anillos nos permite discriminar con mayor rigor si los puntos de arista redondeada son naturales o fueron producidos 


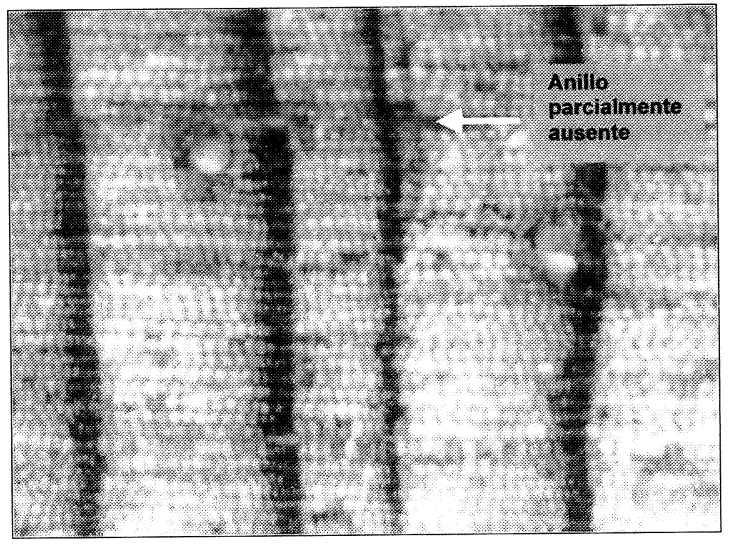

Fig. 7.-Anomalía típica de anillo parcialmente ausente detectada en una de las calas.

por una herramienta de corte. En la figura 8 se observa una inflexión de la secuencia de anillos, debida a la proximidad de un grueso nudo que está asociada a un tramo de rotura de la arista. Si consideramos que los tres puntos detectados con posible gema se encuentran en estas zonas de inflexión de anillos, tenemos base suficiente para rechazar la hipótesis de que estas tres superficies hayan sido producidas por el útil de corte de una manera casual, en total concordancia con los tramos de inflexión de anillos. Se trata, por tanto, de zonas ligeramente rehundidas de la propia superficie del tronco, en las que perduraron restos del último anillo fuera del alcance de los cortes de labrado. En consecuencia, podemos afirmar que el árbol alcanzó la edad de 282-283 años y que la datación del último anillo coincidirá con la fecha de tala del árbol.

\section{Dataciones de carbono-14}

La muestra correspondiente a la cala $\mathrm{C}$ se considera fiable a efectos de datación, una vez eliminada la ligera pudrición verde que afecta a una parte muy pequeña, ocasionada sin duda por la invasión fúngica a favor de una fenda profunda del leño. Al ser ésta la única muestra disponible, se decide medir también el serrín obtenido en la perforación, de modo que pueda servir tanto de control cronológico como de la contaminación debida a la pudrición.

Las dos muestras seleccionadas difieren tanto en cantidad como en la composición de sus anillos (fig. 5):

Muestra tipo cala (12 gramos). Madera sana integrada por 13 anillos que participan desigualmente en la masa total: anillos números 23 a $29(25 \%)$ y anillos números 30 a $35(75 \%)$. El centro de masas se sitúa en torno al anillo $\mathrm{n}^{\circ} 32$. Se procesa toda la madera disponible.

Muestra de serrín (72 gramos). Madera con posible contaminación integrada por 24 anillos del intervalo 19-42, con una distribución de masas uniforme y cuyo centro coincide también con el ani$110 \mathrm{n}^{\circ}$ 32. Dada la abundancia de la muestra se aplicó un procedimiento químico selectivo para aislar la celulosa de los otros componentes de la madera.

En consecuencia, ambas muestras pueden ser referidas al anillo $\mathrm{n}^{\circ} 32$ de la secuencia conjunta $1 \mathrm{~A} /$ $1 \mathrm{~B}$, por lo que a las respectivas determinaciones de edad de carbono-14 se deberán añadir los 250-251 años que medían hasta el último anillo de la viga observado mediante el estudio dendrocronológico.

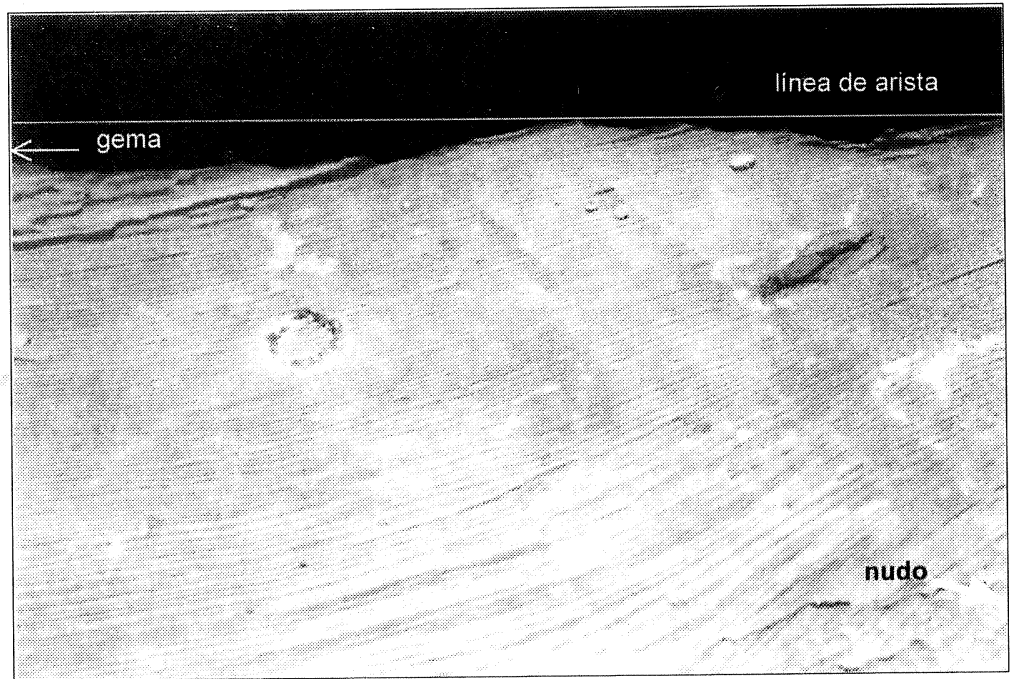

Fig. 8.- Inflexión de la secuencia de anillos, debida a la proximidad de un grueso nudo, en la arista de la viga, indicio de conservación de la gema del tronco. 
Las dos muestras se transforman sucesivamente en dióxido de carbono, acetileno y benceno, siendo su actividad de carbono-14 medida en un contador de centelleo líquido de bajo fondo. Las dataciones que se obtuvieron fueron las siguientes:

\begin{tabular}{|c|c|c|c|c|}
\hline Muestra & Material & Referencia & Edad C-14 años BP & Edad calibrada cal AD \\
\hline cala C & madera & CSIC-1318 & $1880 \pm 29$ & $\begin{array}{c}79-223(100)^{*} \\
71-235(100)^{* *}\end{array}$ \\
\hline serrín & celulosa & CSIC-1323 & $1813 \pm 29$ & $\begin{array}{c}132-260(87)^{*} \\
289-324(13)^{*}\end{array}$ \\
\hline
\end{tabular}

* Programa CALIB 3.03, curva bidecadal, método B, 2 sigma. Entre paréntesis los porcentajes que corresponden a cada intervalo.

** Idénticas condiciones pero con la curva decadal.

Ambas edades carbono-14 parecen confirmar la antigüedad de la viga de madera $\mathrm{y}$, aun cuando son estadísticamente semejantes, la pequeña diferencia entre ellas pudiera reflejar una muy ligera presencia de pudrición en el serrín, haciéndolo algo más moderno. Sin embargo, estas edades de laboratorio no reflejan años reales, por lo que deben calibrarse antes de poder ser utilizadas.

El proceso de calibración es matemáticamente complejo y sólo puede llevarse a cabo mediante programas desarrollados para tal fin (CALIB de la Universidad de Washington, OXCAL de la Universidad de Oxford, etc.; Stuiver y Reimer 1993, Ramsey 1995). Con ellos se convierten las edades convencionales en edades calibradas, cuya escala es ya en años solares o de calendario. El proceso gráfico de calibración para la fecha CSIC-1318 se observa en la figura 9, donde en el eje de ordenadas se representa la curva gaussiana de la edad convencional $1880 \pm 29$ años BP y en el eje de abcisas la edad calibrada (en negro) con una distribución sumamente irregular. La curva de calibración utilizada en este caso para la conversión es la bidecadal y corresponde al tramo comprendido entre 0 y 400 cal AD.

Teniendo en cuenta que el centro de masas para la cala $C$ está en el anillo $n^{\circ} 32$, la edad que realmente interesa desde el punto de vista arqueológico, la del momento en que el árbol fue talado y utilizado, estará comprendida entre los años 330 y 474 de la era, tal como refleja el cuadro siguiente:

\begin{tabular}{|c|c|c|c|}
\hline Referencia & Edad calibrada cal AD & Anillos a sumar & Edad estimada años AD \\
\hline CSIC-1318 & $79-223$ & $283-32=251$ & $330-474$ \\
\hline
\end{tabular}

\section{CONCLUSIONES}

Como hemos visto, hay que considerar el intervalo 330-474 como fecha post quem inmediata a la utilización del árbol. Esta respuesta que los análisis dan a la pregunta sobre la datación no coincide con ninguna de las dos propuestas hipotéticas previas (segunda mitad del s. vII o siglo IX), quedando alejada de ellas, en sus valores medios, al menos 250 y 450 años. Desde el punto de vista de la datación de la iglesia esta contestación merece algún comentario y supone una lección.

La observación inmediata es la larga precedencia temporal de la madera respecto a la construcción del edificio. Por lo tanto debe pensarse si la madera ha sido reutilizada como material de construcción, igual que lo fueron algunos de los sillares (Caballero y Arce: 224-5 y n.3). Los ejemplos de sillería reutilizada de construcciones anteriores son normales en iglesias altomedievales del grupo al que per- tenece La Nave (Caballero et al.: 151-2), por lo que este dato reforzaría la posibilidad de que el material constructivo de La Nave procediera de un edificio anterior desmontado para conseguir material constructivo. Aunque sin ningún tipo de seguridad, puede plantearse que, si la viga perteneció al mismo edificio al que pertenecieron los sillares, el edificio que sirvió de cantera pudo haber sido construido en una fecha en torno al año 400 d.C.

Otro comentario merece el alabeo de la viga, que hubo de ocurrir durante un tiempo muy breve, de pocos años después de su labrado en verde. Al suponer como más cierta la reutilización de la viga, hay que aceptar que en S. Pedro de La Nave se utilizó alabeada y que el alabeo tuvo que ser corregido con la forma de los sillares. Constructivamente esto no parece lógico, pero no se nos ocurre otra posible solución al problema. Si aceptamos los datos de fecha de la corta, el breve lapso de tiempo entre la corta y el alabeo y el largo desfase cronológico en- 


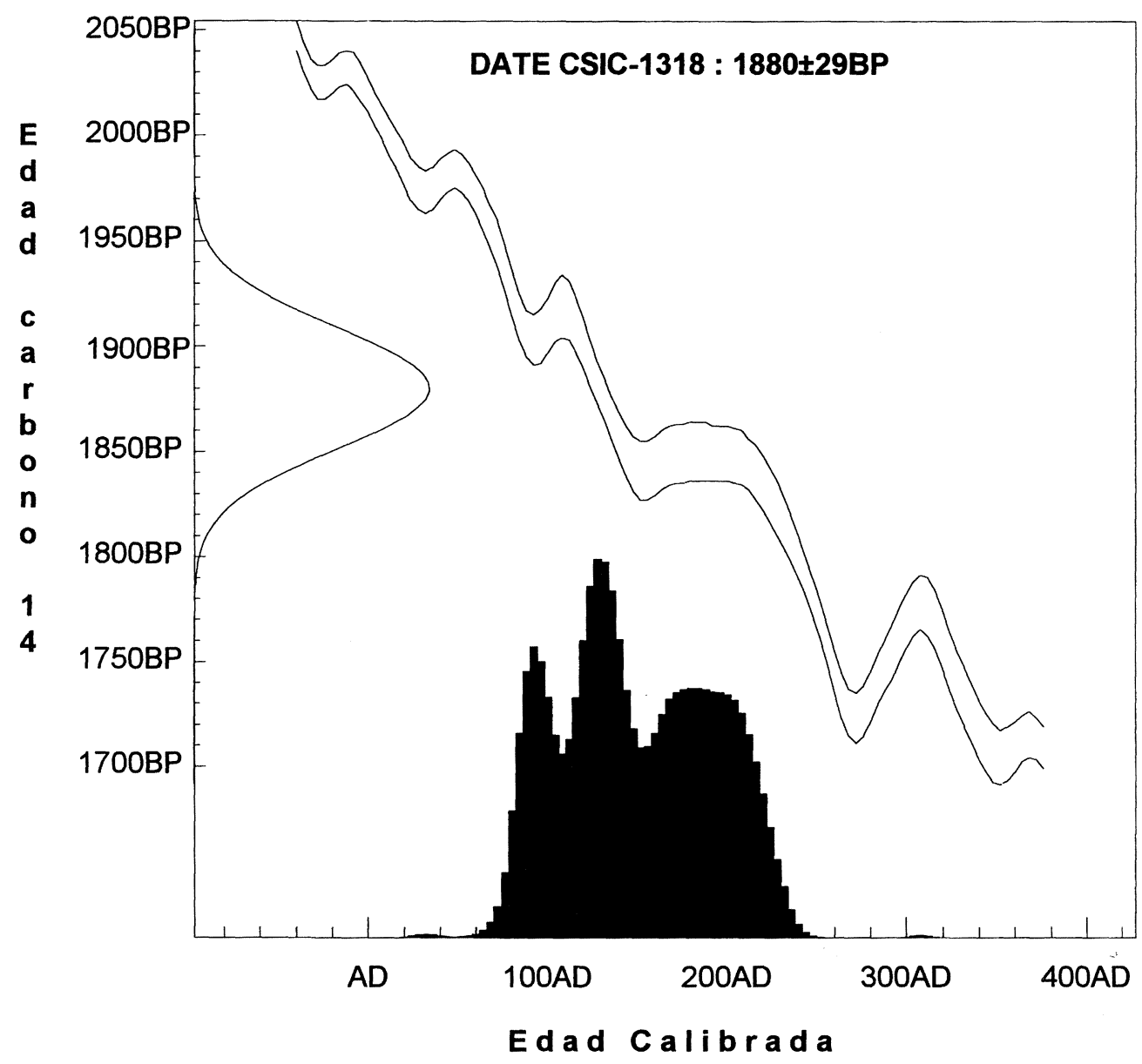

Figura 9.-Calibración de la fecha CSIC-1318. Programa OXCAL, curva bidecadal, método B, 2 sigma.

tre la fecha de la corta y la reutilización en el edificio actual, hay que aceptar como inexcusable que se acoplara la forma de los sillares a la de su alabeo. Hoy no existe posibilidad de comprobar este hecho, que necesitaría desmontar de nuevo el edificio. Pero la talla visible de los sillares, con lechos de hiladas ondulados y juntas oblicuas, permiten aceptar como posible este acople.

La procedencia de la madera no es incompatible con la existencia de un edificio previo cercano al lugar de La Nave. Es más lógica la adquisición de la madera para el primer edificio en un lugar lejano, que no el traslado de la piedra, posiblemente reutilizada, desde un lugar muy alejado del actual lugar de La Nave; aceptando que, en cualquier caso, la piedra se sacó de una cantera cercana, según la observación que efectuó Gómez Moreno (1927: 61).

Después de todas estas matizaciones, el análisis muestra una enseñanza. Si suponemos que en vez de dar la fecha circa 400, su fecha hubiera equivalido a la de la primera de las hipótesis, mediados del s. vII, es posible que hoy estuvieramos considerando la probabilidad de una cronología visigoda para la iglesia de S. Pedro, sin tener en cuenta la relatividad de esta datación, derivada de las limitaciones del análisis y del uso de la viga. Es evidente que para salvar estas limitaciones es necesario conseguir series completas de dataciones de maderas realizadas en parecidas condiciones a las de La Nave, con el control previo de sus variables, especialmente su relación contextual con el edificio (la estratigrafía del edificio) y el análisis formal de la madera, tanto en relación con su uso constructivo, como con su propia constitución. Y de las que se hagan análisis de dendrocronología y de carbono-14 conjuntos.

La existencia de madera en las iglesias altomedievales de la meseta Norte parece que cumple estas condiciones (Caballero y Arce: 269-70): Peñalba y Escalada (León); Baños (Palencia); Fuentearmegil, Gormaz y Berlanga (Soria); Barriosuso 
(Burgos); Tricio y Sta. Coloma (Navarra), etc. Consideremos, por ejemplo, la importancia de dos casos. Las dos grapas de la propia iglesia de La Nave pueden ofrecer una datación por carbono-14 más cercana a la de la iglesia, dado que el corte de la madera para las grapas ofrece más garantía de ser coetánea a la construcción de la iglesia, aunque siempre habrá que tener en cuenta la diferencia entre la fecha de los anillos analizados y la de la gema, equivalente a la del corte real del árbol. También tiene gran interés contrastar la inscripción de la iglesia de S. Juan de Baños, cuya fecha de 652/661 se considera clave para considerar de época visigoda esta serie de iglesias, con la de sus maderas: dos cargaderos de dintel en su puerta; una gruesa viga al parecer aun in situ en su hueco de apeo, en la nave lateral derecha; y uno o dos maderos de zuncho en la fachada Oeste de la nave.

En el caso de La Nave y a la vista de los resultados de edad obtenidos tras la calibración, se considera oportuno realizar en el futuro un nuevo ensayo de datación de carbono-14 a la viga de La Nave, con el fin de lograr una posición más favorable sobre la curva de calibración y, de este modo, mejorar la datación absoluta. La zona que reúne mejores condiciones es el extremo inferior de la viga, realizando la perforación en dirección axial y centrada en la médula del árbol. Complementariamente, será oportuno la determinación de edad por el método del carbono-14 de, cuando menos, una de las grapas de madera conservadas.

\section{BIBLIOGRAFÍA}

AItKEn, M.J. 1990: Science-based dating in archaelogy. Longman. London. 273 pp.

Caballero Zoreda, L. 1994-95: Un canal de transmisión de lo clásico a la alta Edad Media española. Arquitectura y escultura de influjo omeya en la Península Ibérica entre mediados del s. VIII e inicios del s. X, Al-Qantara 15, 321-48 y 16, 107-24.

ID. 1997: Observations on Historiography and Change from the sixth to tenth centuries in the North and West of the Iberian Peninsula, en Díaz-Andreu, M. y Keay, S. (edit.), The Archaeology of Iberia. The Dynamics of Change, Routledge, Londres y Nueva York, 235-264.

ID. y ARCE, F. 1997: La iglesia de San Pedro de La
Nave (Zamora). Arqueología y arquitectura, Archivo Español de Arqueología 70 221-274.

ID. y Cámara Muñoz, L.; Latorre González-Moro, P. y Matesanz Vera, P.: La iglesia prerrománica de S. Pedro el Viejo de Arlanza (Hortigüela, Burgos), Numantia 5 139-165.

Camps Cazorla, E. 1940: El arte hispanovisigodo, en Historia de España, dirigida por Menéndez Pidal, III, Madrid (1963), 598 ss.

Catalán, G. (ed.) 1991: Las regiones de procedencia de Pinus sylvestris L. y Pinus nigra Arn. subsp. salzmannii (Dunal) Franco en España. ICONA. Madrid.

Corzo SÁnchez, R. 1986: San Pedro de La Nave. Estudio histórico y arqueológico de la iglesia visigoda, Zamora.

Eckstein, D.; Baillie, M. G. L. y Egger, H. 1984: Handbook for Archaeologists $N^{\circ} 2$ Dendrochronological Dating, European Science Foundation. Strasbourg, 1, 47 pp.

Gómez Moreno, M. 1927: Catálogo Monumental de España. Provincia de Zamora (1903-1905), Madrid.

ID. 1966: Primicias del arte cristiano español, Archivo Español de Arte 39 101-39.

GRUPPO DI LAVORO PER LA DENDROCRONOLOGIA 1985: Situazione delle cronologie al 1985, Dendrochronologia $3216 \mathrm{pp}$.

HausChiLD, Th. 1972: Westgotische Quaderbauten des 7. Jarhunderts auf der Iberischen Halbinsel, Madrider Mitteilungen, 13 270-85.

Mateos Rodríguez, M. y Esteban Ramírez, L. 1980: San Pedro de La Nave, Zamora.

PARENTI, R. 1988: Sulla posibilità di datazione e di clasificazione delle murature, en Francovich, R. y Parenti, R. (a cura di), Archeologia e restauro dei monumenti (Siena 1987), Florencia, 280304.

RAMSEY, C. B. 1995: Radiocarbon calibration and analysis of stratigraphy: The Oxcal Program, Radiocarbon 37 (2) 425-30.

Stuiver, M. y Reimer, P. J. 1993: Extended ${ }^{14} \mathrm{C}$ data base and revised Calib $3.0{ }^{14} \mathrm{C}$ age calibration program, Radiocarbon 35(1) 215-30.

Torres Balbás, L. 1933: La reparación de los monumentos antiguos en España II, Arquitectura. Colegio Oficial de Arquitectos, XV 129 ss. (Reeditado en Id. 1996: Sobre monumentos y otros escritos, Colegio Oficial de Arquitectos de Madrid, Madrid, 269-304). 